\title{
Los últimos intentos reformadores de la prensa del movimiento (1975-1976)
}

\author{
Dr. Ricardo M. Martín de la Guardia \\ Prof. Titular de Historia de la Prensa \\ Universidad de Valladolid
}

\begin{abstract}
Durante la década de los años setenta la preocupación por la marcha económica de la compleja red de publicaciones que constituía la Prensa del Movimiento comenzó a desplazar incluso a los afanes de control político sobre dichos medios. Los gestores económicos comenzaban a imponer sus criterios sobre la dirección política, más aún cuando en 1975 la muerte del general Franco dejó sin objetivos claros al poderoso aparato propagandístico. Por si fuera poco, las pérdidas continuaban su marcha ascendente, haciendo insostenible la permanencia de la Cadena como hasta entonces.

La necesidad apremiante de mejorar los resultados económicos de las explotaciones pasaba no sólo por aumentar las ventas de ejemplares -lo que justificaba políticamente buena parte de las publicaciones- sino, de manera especial, por incrementar la publicidad contratada, reducir costos en todas las partidas presupuestarias y reestructurar la Delegación Nacional de Prensa.

Nuestro objetivo es, dentro de este contexto crítico, analizar los últimos proyectos reformadores que existieron para dar continuidad a la Prensa del Movimiento entre 1975 y 1976, sobre todo a partir de la llegada de Emilio Romero a la Delegación Nacional. El fin de estos planes renovadores era acabar con la situación lamentable de la economía empresarial, por un lado, y transformar las estructuras heredadas, por otro. Sin embargo, el inicio de la transición política, el nacimiento de nuevos diarios de opinión al amparo de las libertades democráticas y las dificultades para recuperar la credibilidad de muchos de estos medios con una competencia cada dificultades para recuperar la credibilidad de muchos de estos medios con una competencia cada
vez mayor fueron, junto a otras, algunas de las causas que hicieron inviables estos últimos proyectos.
\end{abstract}

\section{La situación de la Cadena en 1975}

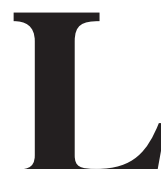

os planes de saneamiento para las explotaciones de la Prensa del Movimiento no eran precisamente desconocidos para los gestores de aquel omplejo organigrama empresarial. En la práctica, ya desde la década de os años cuarenta, bien parcial, bien totalmente, los programas de mejora económica de la Cadena fueron una constante en la evolución de la misma.

Sin embargo, a la altura de 1975, la situación real de los medios de comunicación escritos del Movimiento comenzaba a revestir una gravedad especial. La acumulación de pérdidas de dicha cadena de prensa -a pesar de la rentabilidad innegable de algunos de los diarios-, la escasa o nula incidencia de 
otros muchos a tenor de su limitada tirada, los abultados gastos de personal, en concreto de determinadas plantillas que tenían a todas luces un número excesivo de trabajadores (desde redactores a linotipistas), y, finalmente, la política tradicionalmente seguida desde la Delegación Nacional de Prensa y Radio del Movimiento, orientada a mantener bajo su vigilancia y control la amplia red de publicaciones esparcidas por todo el territorio nacional sin tener en cuenta si resultaban o no rentables, eran hechos contrastados que incidían de manera muy negativa sobre las precarias finanzas de una empresa que debía acudir regularmente a las ayudas estatales para poder subsistir.

Por si fuera poco, según avanzaba la década de los setenta, las transformaciones sociales y la tibia pero progresiva apertura política del país influyó en muchos de estos medios cuya práctica periodística era netamente ideológica, legitimadora del régimen franquista, y que cada vez tenía menor acogida entre una opinión pública que se había desperezado y no mostraba interés por el discurso retórico y efectista, propio todavía de algunas de las «unidades de explotación» -en la jerga oficial al uso- de la Prensa del Movimiento.

De hecho, y como hemos comentado en otro lugar, «un análisis de la situación económica de la cadena de prensa estatal en 1975 mostraba a las claras la inviabilidad de la misma sea cual fuere el punto de referencia que se tomara» ${ }^{1}$. Por ejemplo, la publicidad contratada por los periódicos y revistas del Movimiento totalizó algo más de 1.500 millones de pesetas sobre los 1.650 previstos, lo que significaba un estancamiento cuando no un retroceso efectivo en las ventas por publicidad, una partida clave para la estabilidad económica de la Cadena. Incluso el espacio publicitario insertado fue menor respecto al de 1974, y muy especialmente en el caso de Sur, La Voz de España, Córdoba o El Eco de Canarias. En cambio, el aumento constatado en El Pueblo Gallego, Diario Español, Arriba, La Voz de Almería, Línea y El Telegrama de Melilla, las seis publicaciones con mayor crecimiento de superficie publicitaria contratada en 1975, no alcanzaba entre todas ellas la pérdida sufrida sólo por Sur $(1.415 .497 \mathrm{~mm} \text {. frente a } 2.294 .380 \mathrm{~mm} \text {. })^{2}$.

Los ingresos por venta de publicaciones en 1975 ascendieron a 998,3 millones de pesetas, unos cien millones más que el año anterior, si bien debemos considerar que el precio del ejemplar había pasado de seis pesetas en los tres primeros meses de 1974 a siete pesetas entre mediados de marzo y junio, y a ocho a partir de esta fecha y durante todo 1975. La tirada efectiva media diaria en este

1 MARTín DE LA GUARDIA, Ricardo M., Información y Propaganda en la Prensa del Movimiento. Libertad de Valladolid, 1931-1979, Valladolid, Secretariado de Publicaciones de la Universidad, 1994, pág. 313. «El porcentaje del espacio publicitario sobre el espacio total publicado alcanzó en 1975 el 21,80\% «El porcentaje del espacio publicitario sobre el espacio total publicado alcanzó en 1975 el $21,80 \%$
disminuyendo en un $3,2 \%$ respecto al año 1974». Informe Económico. Año 1975. Delegación Nacional de disminuyendo en un $3,2 \%$ respecto al año 1974». Informe Económico. Año 1975. Delegación Nacional de
Prensa y radio del Movimiento. Departamento Económico-Administrativo. Sección Prensa. (A)rchivo Prensa y radio del Movimiento. Departamento Económico-Administrativo. Sección Prensa. (A)rchivo
(G)eneral de la (A)dministración. (M)edios de (C)omunicación (S)ocial del (E)stado. Director Económico(G)eneral de la (A)dministra 
año para los diarios del Movimiento fue de 462.083 ejemplares, también mayor que la de 1974. Los aumentos más significativos fueron los de Marca (5.034 ejemplares diarios más), Arriba (4.180) y Levante (4.153). Estas mejoras eran, sin embargo, únicamente aparentes. Los ingresos brutos en 1975 habían superado los 2.800 millones de pesetas, unos 90 millones más que en el ejercicio anterior, pero 85 millones menos de lo presupuestado. En cambio, los gastos ascendieron a 3.660 millones, casi 860 millones más que el año anterior y 315 millones más de lo previsto. La cifra era desorbitada, sobre todo en algunos gastos específicos:

«En el año 1974 la suma de los gastos de personal y consumos representa el 74,40\% de los ingresos típicos. En 1975 estas dos partidas de gasto absorben el $100,57 \%$ de los ingresos típicos (se refiere a los ingresos de publicidad más venta de prensa).»³

El resultado de la explotación era, por tanto, desolador. Si en 1974 el déficit rondó los 75 millones de pesetas, en el ejercicio de 1975 se disparó hasta los 842 millones, es decir, 400 millones más de lo presupuestado. Esta desviación negativa era consecuencia en un 89,38\% del incremento en los gastos de personal (casi 245 millones más de lo previsto), como ya hemos comentado, y de la disminución de la publicidad (113 millones menos)

\section{El Proyecto de Reforma de 1975}

Ante esta situación, las autoridades reaccionaron por fin con cierta contundencia. En febrero de 1975, Emilio Romero, un periodista muy activo y con larga experiencia, era designado nuevo Delegado Nacional. Pronto, respaldado por Francisco Soler Valero, nombrado director Económico-Administrativo de la Prensa del Movimiento, pergeñó un vasto plan de transformaciones estructurales que hiciera posible el mantenimiento económico de los medios estatales y los mejorara en calidad y difusión. El plan resultaba un ejemplo cabal de aunar medidas reformistas muy novedosas por lo radicales con otras ya conocidas por haber sido experimentadas pero que, o bien habían resultado fallidas, o bien no se habían podido llevar a la práctica en su totalidad. En cualquier caso, este amplio ensayo reformistas mostraba que «aunque los aspectos políticos y organizativos siguen estando presentes es, no obstante, la necesaria adecuación a las nuevas necesidades y circunstancias la que tildará desde una vertiente económica las principales inquietudes de la Cadena y de sus responsables en los últimos años del franquismo» ${ }^{4}$.

3 Ibídem, pág. 5.

4 MONTABES PEREIRA, Juan, La prensa del Estado durante la transición política española, Madrid, C.I.S., 1989,pág.37. 
Era éste, en efecto, un cambio sustancial. Los numerosísimos informes, análisis y estudios de todo tipo elevados durante décadas a las autoridades competentes por los expertos económicos de la Prensa del Movimiento habían chocado en muchas ocasiones con la primacía que estas últimas daban al objetivo políticoideológico de las publicaciones del Estado, el cual siempre primó sobre cualquier otra consideración, lo que había frenado, cuando no paralizado totalmente, numerosos programas de reforma. Por fin se iba a acometer una renovación económicofinanciera sin las cortapisas impuestas desde la esfera política.

A partir de los análisis realizados por los órganos pertinentes en la Delegación, la finalidad prioritaria consistía en recortar gastos por todos los medios posibles $^{5}$. En un primer lugar, era evidente la hipertrofia de la plantilla existente, no sólo la derivada del personal fijo, sino el exceso de horas extraordinarias, plazas interinas, suplencias, etc. que, sin revertir en la productividad, provocaban un aumento desmesurado de los gastos. Calculaba el informe que la racionalización de los puestos de trabajo, reestructurando y adaptando las plantillas, y eliminando los citados excesos, supondría un menor coste anual en 1976 de 120 millones de pesetas. Una Junta de Admisión de Personal designada por la Delegación consideraría los traslados de personal de unos periódicos a otros cuando lo estimara oportuno ${ }^{6}$.

Así mismo en mayo de 1975, la Cadena había iniciado un programa de jubilaciones que alcanzaba a 200 trabajadores de los distintos niveles (desde redactores hasta linotipistas) con un ahorro previsto para 1976 de 70 millones de pesetas. Junto a este programa, y ya en 1974, los responsables de la Cadena habían puesto en marcha un plan de regulación de empleo, que se pretendía reforzar en 1975 y 1976. Una vez consultado con el Ministerio de Trabajo para evitar cualquier tipo de conflicto sindical, los expedientes de regulación iban a afectar fundamentalmente a los trabajadores mayores de sesenta años. Quienes se acogieran al plan podrían retirarse con la totalidad del sueldo mientras que sus plazas quedarían amortizadas:

«La política de jubilaciones habrá de acelerarse con la supresión inmediata de los cinco periódicos previstos (se refiere a Amanecer, Diario de Cuenca, La Voz de Castilla, La Prensa y Libertad) ya que supone la posibilidad de forzar más, en algún periódico, las jubilaciones, al contar con personal trasladado procedente de las unidades de explotación que han de cerrarse»?

5 Esquema de acciones para 1976. Programa de explotación (a corto y medio plazo). (A)rchivo del (M)inisterio de (C)ultura. Caja $\mathrm{n}^{\circ} 75.689$

Nota informativa de medidas a tomar y en marcha, para la reestructuración de la Cadena de Prensa en el próximo ejercicio, 1976. Madrid, 26 de diciembre de 1975, pág. 2. En ibídem.

7 Anexo $\mathrm{n}^{3} 3$, en ibídem. 
Entre 1974 y 1975, 218 empleados de la Prensa del Movimiento se habían jubilado conforme a este plan, y si los cálculos de la Delegación eran correctos, otros 350 lo harían en 1976, con una economía en este año y por dicho concepto de 122 millones de pesetas 8 .

En segundo término, y como complemento a las medidas sobre plantillas, el cierre de ciertos diarios cuyas pérdidas económicas eran exageradas aparecía como otro de los puntos capitales del plan. Según Begoña Zalbidea, Emilio Romero tenía en mente suspender doce publicaciones, pero los numerosos problemas que se le presentaron para llevar a cabo una medida tan extrema cercenaron esta iniciativa9. Los matutinos Amanecer de Zaragoza, Diario de Cuenca y el burgalés La Voz de Castilla deberían desaparecer definitivamente a partir del 1 de febrero de 1976, lo mismo que los vespertinos La Prensa de Barcelona y Libertad de Valladolid, si bien aquí con alguna particularidad. En cuanto al primero, el mando nacional entendía que con mantener en la Ciudad Condal el ya de por sí ruinoso Solidaridad Nacional era suficiente siempre y cuando fuera diseñada una profunda remodelación para este periódico. En el caso del veterano «diario nacional-sindicalista» vallisoletano, éste aparecería renovado y potenciado como diario de ámbito regional para Castilla. A tenor del fracaso económico y de la exigua tirada de los órganos informativos del Movimiento en Zamora, Burgos y el propio Valladolid, el nuevo proyecto debería cubrir el área informativa castellana, otorgando mayor peso a las noticias locales con el objetivo de ganar lectores tanto a la prensa privada provincial como a los poderosos diarios nacionales editados en Madrid. La cancelación de las cinco publicaciones conllevaría un ahorro de 145,5 millones de pesetas en 1976.

Sin embargo, este programa de cierres no pudo llevarse a cabo tal como estaba diseñado. La Delegación Nacional tuvo ya serias dificultades para integrar en febrero de 1975 Voluntad de Gijón en La Nueva España de Oviedo, uno de los diarios más rentables de la Cadena, y hacer una operación parecida un año después con la desaparición de Jornada en beneficio de Levante. Política que provocó muchas reticencias cuando también en febrero de 1976 se decidía poner fin a la salida diaria de tres publicaciones: el periódico navarro fundado por Fermín Izurdiaga, Arriba España, así como el vespertina malagueño La Tarde y el burgalés La Voz de Castilla. Arriba España constituía uno de los fenómenos más espectaculares del desastre económico de algunos medios estatales: con 426

8 La supresión del coste originado por las situaciones especiales de las que se beneficiaban algunos directores, redactores-jefe, administradores e inspectores de la Prensa del Movimiento se cifraba para 1976 en unos 20 millones de pesetas. Nota informativa..., pág. 2 y anexo $\mathrm{n}^{\circ} 2$. En ibídem.)

9 «Prensa del Movimiento: los pasos contados hacia el aperturismo», en ALVAREZ, Jesús Timoteo (et al.) «Prensa del Movimiento: los pasos contados hacia el aperturismo», en ALVAREZ, Jesús Timoteo (et al. Barcelona, Ariel, 1990, págs. 256-257. 
ejemplares de venta diaria media en 1974 tenía presupuestado un déficit para dicho año de 10,6 millones de pesetas ${ }^{10}$.

Por otra parte, los diarios Baleares, Sevilla, Línea de Murcia, Información de Alicante y $\mathrm{La} \mathrm{Voz} \mathrm{del} \mathrm{Sur} \mathrm{estaban} \mathrm{ya} \mathrm{inmersos} \mathrm{en} \mathrm{un} \mathrm{plan} \mathrm{de} \mathrm{reforma} \mathrm{que}$ incluía la mejora infraestructural y la adecuación de los puestos de trabajo a las necesidades reales. La inversión estaba valorada en unos 340 millones de pese$\operatorname{tas}^{11}$. Proyectos parecidos estaban pensados a medio plazo para reactivar Mediterráneo de Castellón, Odiel de Huelva, El Pueblo Gallego de Vigo, y Patria de Granada $^{12}$.

Finalmente, la revista taurina El Ruedo pasaría a ser editada por Toydesa y 7 Fechas, edición europea, por el Instituto Español de Emigración, con un ahorro conjunto para 1976 de unos 29 millones de pesetas.

La reducción de gastos debía pasar también por economizar el montante destinado a papel-prensa, que era muy elevado al gestionar la Delegación casi cincuenta publicaciones. De ahí que la disminución del gasto de papel y la apuesta por racionalizar su distribución entre los distintos medios según el número de páginas, formato, etc., hubiera sido desde años atrás otra preocupación constante para las autoridades de la Cadena y, a pesar de que algunas medidas se habían tomado en la década de los sesenta fundamentalmente, la confusión fue la tónica general. Así, algunos periódicos con pérdidas económicas elevadas, escasa contratación publicitaria y difusión mínima, ofrecían día a día al lector un número excesivo de páginas si se comparaba con otros más saneados y con posibilidades reales de ampliar su mercado. Era el caso, por ejemplo, del Diario Español de Tarragona, El Eco de Canarias o Solidaridad Nacional de Barcelona que, con tiradas inferiores a 7.000 ejemplares, salían a la calle con una media de 30 y 32 páginas, mientras que Levante o La Nueva España, rentables en aquellos años, lo hacían con 36 ó 32, respectivamente. De este hecho se derivaba un gasto superfluo de papel así como la necesidad de ajustar tiradas, formato y número de páginas a la capacidad real de venta (tanto de publicidad como de ejemplares) para frenar el enorme volumen de devoluciones producidas:

10 Dichos datos, respectivamente, en Venta media diaria de periódicos del año 1966 al año 1976. Delegación Nacional de Prensa y Radio del Movimiento. Departamento Económico-Administrativo. A.G.A., M.C.S.E Archivo General Caja $\mathrm{n}^{\circ} 172$ y Presupuestos año 1974. A.G.A. M.C.S.E. Patrimonio, Caja no 148. Si embargo, otros como Amanecer o La Prensa continuarían acumulando pérdidas hasta que en julio de 1979 fueran clausurados definitivamente.

11 Programa de actuación para el Ejercicio 1976. Sector Prensa, pág. 2. Delegación Nacional de Prensa y Radio del Movimiento. Departamento Económico-Administrativo. A.M.C. Caja $\mathrm{n}^{\circ} 75.689$.

12 Aunque no había nada concreto al respecto, uno de los informes comentaba: «Es preocupación constante Aunque no había nada concreto al respecto, uno de los informes comentaba: «Es preocupación constante de este Departamento Económico-Administrativo el establecer todas las premisas que llevan a una eficaz administración y rentabilidad de las siguientes explotaciones: La Nueva España, Información, Sur, Los 1976 ... pág. 3. En ibídem. 
«A partir del 1 de enero de 1976 y para el primer semestre se establece reducción de páginas, en base a: formato, porcentaje, publicidad, competencia, área de difusión y promoción. El ahorro año, en el consumo de papel prensa por esta medida se valora en 58 millones de pts.» ${ }^{13}$

El plan establecía una segunda fase durante el segundo semestre de 1976 en la cual los órganos nacionales elaborarían proyectos específicos para cada publicación donde se establecería la paginación más adecuada e, incluso, cabría la posibilidad de pergeñar una maqueta tipo de cada periódico de la Cadena.

Como podemos observar en el Anexo 1, el número de páginas debía reducirse en todos los periódicos -excepto El Telegrama de Melilla que contaba sólo con ocho y La Voz del Sur con doce- para, con posterioridad, valorar el previsible aumento de superficie publicitaria y volver a adecuar la paginación a esta nueva realidad.

Finalmente, entraba dentro de las previsiones de la Delegación la enajenación de algunos inmuebles ocupados por diarios del Movimiento, bien por cierre de éstos, bien por cambio de ubicación. Sería el caso de los locales de Málaga, Córdoba, Alicante, Gijón, Jerez de la Frontera y Zaragoza, cuyo valor conjunto rondaba los 133 millones $^{14}$.

Como era lógico, el diseño del plan no contemplaba solamente la reducción y el control exhaustivo sobre los gastos, sino la mejora en la partida de ingresos. El aumento de la contratación publicitaria, auténtica obsesión de los gestores económicos de la Cadena desde el nacimiento de ésta, constituía una pieza clave para el saneamiento financiero de la misma. El 28 de mayo de 1975, el Delegado Nacional daba el visto bueno a un proyecto presentado por la Dirección Económico-Administrativa de la Prensa del Movimiento, por el cual se constituía una Gerencia Comercial con rango de Dirección Orgánica. Con la seguridad, como hemos dicho, de que cualquier tipo de solución económica debía pasar por un aumento de la publicidad contratada, además por supuesto de la venta de ejemplares, se reestructuraban los servicios hasta entonces existentes y se adscribían a esta Gerencia los siguientes:

«El Primer Servicio de Promoción de Prensa tiene como misión estimular la compra de diarios y fomentar el número de suscriptores y perfeccionar los

13 Nota informativa..., pág. 1. En ibídem.

14 «El camino emprendido dio también un impulso fuerte a una actuación que había comenzado unos años antes y que se extendería hasta 1978, por lo menos: la venta de una buena parte de los terrenos ocupados por los periódicos estatales (generalmente céntricos) y su traslado a la periferia de las ciudades, incluidos os "polígonos de desarrollo", en una importante operación especulativa que comprendía, a veces, la adquisición de nueva maquinaria y la correspondiente venta de la antigua». ZALBIDEA, B,. op. cit., pág. 
servicios de distribución para lograr una oportuna difusión, con objeto de aumentar la tirada y crear un estrato idóneo para que al amparo de una superior tirada se posibilite una mayor demanda publicitaria.

«El Segundo Servicio de Promoción de Publicidad de Prensa y Radio tiene como principal gestión fomentar la demanda publicitaria (...) perfeccionando los canales de oferta y ayudando a nuestros medios en la consecución de las campañas publicitarias y sobre todo conseguir anuncios de tipo nacional y regional (...) estableciendo un contacto directo en las agencias y anunciantes (...).

«Al Tercer Servicio [Coordinación Comercial] se le ha encomendado la Coordinación de toda la acción comercial de la Gerencia, preparando los supuestos necesarios para la toma de decisiones, mediante informes, estudios, análisis etc.» ${ }^{15}$.

Junto a esta importante remodelación en los órganos nacionales, se querían potenciar las funciones de la persona o del equipo encargado de promocionar la venta publicitaria en cada una de las unidades de explotación, para lo cual debía estar altamente cualificado con el fin de cumplir las esperanzas puestas en la mejora de los ingresos por esta partida. Los informes hasta ahora citados estimaban que la venta bruta por publicidad en 1976 aumentaría un 10\% para toda la Cadena, aproximadamente unos 100 millones de pesetas.

Bastante optimistas eran también los cálculos realizados sobre la venta de prensa. En teoría, y tal como pensaba la Delegación, si el producto final -el ejemplar diario- había mejorado en calidad (una vez suprimidos los no rentables y reestructurados los demás), el público lector se acercaría otra vez a los diarios del Movimiento. Si bien en los tres primeros meses de 1975 se vendieron 40.000 ejemplares más que en el mismo período de 1974, lo cierto es que el aumento previsto no era notable y además estaba concentrado en unos pocos diarios que eran los más vendidos tradicionalmente dentro de la Prensa del Movimiento ${ }^{16}$.

En cuanto a la agencia PYRESA, con un déficit estimado de 81 millones para 1976, la solución alcanzada por los órganos competentes no era muy imaginativa: recurrir a una subvención del Consejo Nacional del Movimiento entre 50 y 60 millones de pesetas que al menos sirviera para eliminar los ahogos más inmediatos. También el plan contemplaba una ayuda a fondo perdido del Ministerio de Información y Turismo por valor de 50 millones a la vez que contaba con

15 Circular de Gerencia comercial a Directores y Administradores de la Prensa del Movimiento. 13 de agosto de 1975. A.G.A., M.C.S.E. Caja ${ }^{\circ}{ }^{188}$. Carpeta circulares a diarios.

6 La Delegación Nacional preveía por «mayores beneficios, explotaciones rentables y óptima comercialización, 90 mill./pts.» Nota Informativa de medidas... pág. 2, A.M.C., Caja 75.689. 
que otros 43 del presupuesto de la Delegación Nacional fueran sufragados por la Secretaría General del Movimiento.

$\mathrm{Si}$ hacemos un resumen de las valoraciones hechas por la Delegación Nacional, el resultado podría ser éste:

\begin{tabular}{|l|c|}
\hline \multicolumn{1}{|c|}{ Concepto } & Millones de pesetas \\
\hline Ahorro & 549.5 \\
Subvenciones y recursos & 243.0 \\
& 792.5 \\
Enajenaciones previstas & 132.5 \\
\hline
\end{tabular}

Sin duda, y a tenor de lo que significaban estas cifras, las acciones puestas en marcha a partir del 1 de enero de 1976 para sanear la prensa estatal eran contundentes. Además de la reducción de páginas en las explotaciones de la Cadena y el consiguiente menor gasto de papel, las medidas continuaban y aceleraban el plan de jubilaciones y la reacomodación de los puestos de trabajo, sin olvidar los expedientes de regulación de empleo en aquellos medios en que se estimara necesario.

En el mes de febrero comenzó el cierre definitivo de algunos diarios, la venta de inmuebles, y el recurso a las subvenciones económicas del Ministerio y la Delegación, que debían hacer otro tanto en la mejora futura. Por supuesto, el aumento de ingresos publicitarios y por mayor venta de números diarios daría el empuje definitivo a la consolidación de las publicaciones estatales en el nuevo régimen que se perfilaba en España tras la muerte de Franco.

Sin embargo, el gran plan transformador resultó tardío; algunas de sus disposiciones, como hemos comentado con anterioridad, no se llevaron a la práctica y las previsiones de mejora en determinadas partidas fueron completamente erróneas. Los periódicos del Movimiento perdieron en 1976 cerca de 172,5 millones de pesetas, un 36\% más que en 1975. Los resultados, en comparación con lo presupuestado para 1976, mostraban una desviación negativa de 136,6 millones, de los cuales 41,3 eran menores ingresos y 95,3 mayores gastos ${ }^{17}$. La pérdida acumulada durante 1976 fue de 914,3 millones, mientras que un año antes había alcanzado los 841,8 millones. Aunque el incremento de la misma era aparentemente de 72,5, la realidad era más grave: los 34 millones de pesetas que figuraban en los presupuestos de 1975 como ingresos a cargo de la Secretaría General habían

17 Situación Económica. Delegación Nacional de Prensa y Radio del Movimiento. Departamento EconómicoAdministrativo. Sector Prensa. 1976, pág. 14 A.G.A., M.C.S.E. Director Económico-Administrativo. Caja $\mathrm{n}^{\circ} 46$. 
ascendido a casi 230, de tal manera que el aumento del déficit real era de 267,8 millones ${ }^{18}$.

Las cifras corroboraban la imposibilidad de salir de la crisis no sólo ya por el hecho de no conseguir más publicidad o de mejorar los ingresos por ventas -que descendieron efectivamente en 1976, lo cual mostraba en algunos casos tiradas efectivas ridículas, tal como podemos observar en el Anexo II- sino por la absoluta desconexión entre la mayor parte de los medios del Estado y el público lector. La progresiva ampliación de la oferta de prensa (entre otros, en abril de 1976 aparecía El País y en octubre del mismo año Diario 16) y los déficits todavía más abultados a partir de 1976 pusieron a la antigua Prensa del Movimiento en el camino hacia su desaparición.

18 Ibídem. Según MONTABES PEREIRA, la Cadena tenía un déficit en 1974 de 74.309.865, cifra que aumentaría a 841.774 .535 al año siguiente. Op. cit., pág. 38. También sin citar fuentes concretas, Enrique BUSTAMANTE comenta: «Según un estudio difundido por los propios trabajadores en 1977-78, lo diarios habrían obtenido beneficios en su conjunto hasta 1974, pasando a partir de entonces a acumular pérdidas anualmente, 363 millones en 1975, 540 en 1976, unos 900 en 1977» Los amos de la información
en España, Barcelona, Akal, 1982, pág. 201. 
ANEXO I

\begin{tabular}{|c|c|c|c|c|}
\hline Publicaciones & $\begin{array}{l}\text { Paginación } \\
\text { Ideal }\end{array}$ & \begin{tabular}{c|} 
Media tres \\
trimestres 1975 \\
\end{tabular} & Desviaciones & Observaciones \\
\hline ALERTA & 32 & 36 & -4 & \\
\hline AMANECER & 14 & 16 & -2 & Formato grande \\
\hline ARRIBA & 50 & 54 & -4 & \\
\hline BALEARES & 38 & 42 & -4 & \\
\hline CÓRDOBA & 22 & 24 & -2 & $\begin{array}{l}\text { Lleva paginación } \\
\text { mínima }\end{array}$ \\
\hline DIARIO DE CUENCA & 14 & 16 & -2 & \\
\hline DIARIO ESPAÑOL & 26 & 30 & -4 & \\
\hline EL CORREO DE ZAMORA & 10 & 12 & -2 & Formato grande \\
\hline EL ECO DE CANARIAS & 28 & 32 & -4 & \\
\hline EL PUEBLO GALLEGO & 20 & 22 & -2 & Formato grande \\
\hline EL TELEGRAMA DE MELILLA & 8 & 8 & & $\begin{array}{l}\text { Lleva paginación } \\
\text { mínima }\end{array}$ \\
\hline HIERRO & 16 & 18 & -2 & Formato grande \\
\hline INFORMACIÓN & 36 & 40 & -4 & \\
\hline JAÉN & 24 & 28 & -4 & \\
\hline LA GACETA REGIONAL & 24 & 28 & -4 & \\
\hline LA HORA LEONESA & 24 & 24 & & $\begin{array}{l}\text { Cambio formato } \\
\text { y competencia }\end{array}$ \\
\hline LA MAÑANA & 26 & 28 & -2 & Competencia \\
\hline LA NUEVA ESPAÑA & 30 & 32 & -2 & $\begin{array}{l}\text { Competencia. } \\
\text { Páginas para Gijón } \\
\text { y \% publicidad } \\
\text { elevada }\end{array}$ \\
\hline LA PRENSA & 26 & 30 & -4 & \\
\hline LA VOZ DE ALMERÍA & 24 & 26 & -2 & $\begin{array}{l}\text { Promoción y alto } \\
\% \text { de publicidad }\end{array}$ \\
\hline LA VOZ DE CASTILLA & 24 & 28 & -4 & \\
\hline LA VOZ DE ESPAÑA & 32 & 36 & -4 & \\
\hline LA VOZ DEL SUR & 12 & 12 & & Paginación mínima \\
\hline LEVANTE & 34 & 36 & -2 & $\begin{array}{l}\text { Competencia y } \\
\% \text { de publicidad }\end{array}$ \\
\hline LIBERTAD & 20 & 24 & -4 & \\
\hline LÍNEA & 32 & 36 & -4 & Competencia \\
\hline LOS SITIOS & 14 & 16 & -2 & Formato grande \\
\hline MARCA & 30 & 32 & -2 & $\begin{array}{l}\text { Promoción y } \\
\text { competencia }\end{array}$ \\
\hline MEDITERRÁNEO & 14 & 16 & -2 & Formato grande \\
\hline NUEVA ESPAÑA & 14 & 16 & -2 & Formato grande \\
\hline ODIEL & 14 & 16 & -2 & Formato grande \\
\hline $\begin{array}{l}\text { PATRIA } \\
\text { SEVIIIA }\end{array}$ & 14 & 16 & -2 & $\begin{array}{l}\text { Formato grande } \\
\text { y competencia }\end{array}$ \\
\hline $\begin{array}{l}\text { SEVILLA } \\
\text { SOIIDABIDAD NACIONAI }\end{array}$ & $\frac{14}{28}$ & $\frac{16}{32}$ & $\frac{-2}{-4}$ & Formato grande \\
\hline SOLIDARIDAD NACIONAL & 28 & 32 & -4 & \\
\hline SUR & 40 & 44 & -4 & \\
\hline UNIDAD & 16 & 20 & -4 & \\
\hline
\end{tabular}

Fuente: Nota informativa de medidas a tomar y en marcha, para la reestructuración de la Cadena de Prensa en el próximo ejercicio, 1976. A.M.C. Caja no 75.689. 
180 Los últimos intentos reformadores de la prensa del movimiento (1975-1976)

ANEXO II

Promedio anual de la tirada media efectiva diaria, correspondiente al año de 1976

\begin{tabular}{|c|c|}
\hline DIARIOS & EJEMPLARES \\
\hline ALERTA & 28.104 \\
\hline AMANECER & 2.223 \\
\hline ARRIBA & 14.559 \\
\hline BALEARES & 18.603 \\
\hline CÓRDOBA & 8.172 \\
\hline DIARIO DE CUENCA & 1.573 \\
\hline DIARIO ESPAÑOL & 7.337 \\
\hline EL CORREO DE ZAMORA & 4.459 \\
\hline EL ECO DE CANARIAS & 6.392 \\
\hline EL PUEBLO GALLEGO & 3.663 \\
\hline EL TELEGRAMA DE MELILLA & 1.182 \\
\hline HIERRO & 5.870 \\
\hline INFORMACIÓN & 20.678 \\
\hline JAÉN & 5.146 \\
\hline LA GACETA REGIONAL & 5.703 \\
\hline LA HORA LEONESA & 8.410 \\
\hline LA MAÑANA & 6.692 \\
\hline LA NUEVA ESPAÑA & 39.682 \\
\hline LA PRENSA & 2.068 \\
\hline LA VOZ DE ALMERÍA & 5.936 \\
\hline LA VOZ DE CASTILLA & 1.710 \\
\hline LA VOZ DE ESPAÑA & 34.704 \\
\hline LA VOZ DEL SUR & 4.708 \\
\hline LEVANTE & 39.157 \\
\hline LIBERTAD & 994 \\
\hline LÍNEA & 5.625 \\
\hline LOS SITIOS & 6.934 \\
\hline MARCA & 91.036 \\
\hline MEDITERRÁNEO & 5.874 \\
\hline NUEVA ESPAÑA & 3.372 \\
\hline ODIEL & 5.059 \\
\hline PATRIA & 2.902 \\
\hline SEVILLA & 2.840 \\
\hline SOLIDARIDAD NACIONAL & 3.630 \\
\hline SUR & 23.953 \\
\hline UNIDAD & 9.720 \\
\hline TOTAL DIARIOS & 441.952 \\
\hline SEMANARIOS & EJEMPLARES \\
\hline HOJA DEL LUNES/ALICANTE & 37.944 \\
\hline HOJA DEL LUNES/LAS PALMAS & 20.208 \\
\hline 7 FECHAS/ALEMANIA & 21.448 \\
\hline TOTAL SEMANARIOS & 79.600 \\
\hline TOTAL GENERAL & 521.552 \\
\hline
\end{tabular}

Fuente: A.G.A., M.C.S.E. Análisis de gestión. Caja nº 160. 